\title{
Analysis of dynamic and widespread IncRNA and miRNA expression in fetal sheep skeletal muscle
}

\begin{abstract}
Chao Yuan ${ }^{1}$, Ke Zhang $^{2}$, Yaojing Yue ${ }^{1}$, Tingting Guo ${ }^{1}$, Jianbin Liu ${ }^{1}$, Chune Niu ${ }^{1}$, Xiaoping Sun $^{1}$, Ruilin Feng ${ }^{1}$, Xiaolong Wang ${ }^{2}$, Bohui Yang ${ }^{\text {Corresp. } 1}$

${ }^{1}$ Sheep Breeding Engineering Technology Research Center, Lanzhou Institute of Husbandry and Pharmaceutical Sciences of Chinese Academy of Agricultural Sciences, Lanzhou, Gansu, China

${ }^{2}$ College of Animal Science and Technology, Northwest A\&F University, Yangling, Shaanxi, China
\end{abstract}

Corresponding Author: Bohui Yang

Email address: yangbohui@caas.cn

The sheep is an economically important animal, and there is currently a major focus on improving its meat quality through breeding. There are variations in the growth regulation mechanisms of different sheep breeds, making fundamental research on skeletal muscle growth essential in understanding the regulation of (thus far) unknown genes. Skeletal muscle development is a complex biological process regulated by numerous genes and non-coding RNAs, including microRNAs (miRNAs) and long non-coding RNAs (IncRNAs). In this study, we used deep sequencing data from sheep longissimus dorsi (LD) muscles sampled at day 60,90 , and 120 of gestation, as well as at day 0 and 360 following birth, to identify and examine the IncRNA and miRNA temporal expression profiles that regulate sheep skeletal myogenesis. We stained LD muscles using histological sections to analyse the area and circumference of muscle fibers from the embryonic to postnatal development stages. Our results showed that embryonic skeletal muscle growth can be characterized by time. We obtained a total of 694 different IncRNAs and compared the differential expression between the E60 vs. E90, E90 vs. E120, E120 vs. D0, and D0 vs. D360 IncRNA and gene samples. Of the total 701 known sheep miRNAs we detected, the following showed a wide range of expression during the embryonic stage: miR-2387, miR-105, miR-767, miR-432, and miR-433. We propose that the detected IncRNA expression was time-specific during the gestational and postnatal stages. GO and KEGG analyses of the genes targeted by different miRNAs and IncRNAs revealed that these significantly enriched processes and pathways were consistent with skeletal muscle development over time across all sampled stages. We found four visual IncRNA-gene regulatory networks that can be used to explore the function of IncRNAs in sheep and may be valuable in helping improve muscle growth. This study also describes the function of several IncRNAs that interact with miRNAs to regulate myogenic differentiation. 
1 Analysis of dynamic and widespread IncRNA and miRNA expression in fetal sheep skeletal muscle

2 Chao Yuan ${ }^{1}$, Ke Zhang ${ }^{2}$, Yaojing Yue ${ }^{1}$, Tingting Guo ${ }^{1}$, Jianbin Liu ${ }^{1}$, Chune Niu${ }^{1}$, Xiaopin Sun ${ }^{1}$, Ruilin Feng ${ }^{1}$,

3 Xiaolong Wang ${ }^{2}$, Bohui Yang ${ }^{1 *}$

4

$5 \quad{ }^{1}$ Sheep Breeding Engineering Technology Research Center, Lanzhou Institute of Husbandry and

6 Pharmaceutical Sciences of Chinese Academy of Agricultural Sciences, Lanzhou, P. R. China

$7 \quad{ }^{2}$ College of Animal Science and Technology, Northwest A\&F University, Yangling, P. R. China

8 *Corresponding author:

9 Bohui Yang

10 E-mail: yangbohui@caas.cn

11 Running title: LncRNA expression in fetal sheep muscle 
13

14

\section{Abstract}

The sheep is an economically important animal, and there is currently a major focus on improving its meat quality through breeding. There are variations in the growth regulation mechanisms of different sheep breeds, making fundamental research on skeletal muscle growth essential in understanding the regulation of (thus far) unknown genes. Skeletal muscle development is a complex biological process regulated by numerous genes and non-coding RNAs, including microRNAs (miRNAs) and long non-coding RNAs (lncRNAs). In this study, we used deep sequencing data from sheep longissimus dorsi (LD) muscles sampled at day 60, 90, and 120 of gestation, as well as at day 0 and 360 following birth, to identify and examine the lncRNA and miRNA temporal expression profiles that regulate sheep skeletal myogenesis. We stained LD muscles using histological sections to analyse the area and circumference of muscle fibers from the embryonic to postnatal development stages. Our results showed that embryonic skeletal muscle growth can be characterized by time. We obtained a total of 694 different lncRNAs and compared the differential expression between the E60 vs. E90, E90 vs. E120, E120 vs. D0, and D0 vs. D360 lncRNA and gene samples. Of the total 701 known sheep miRNAs we detected, the following showed a wide range of expression during the embryonic stage: miR2387, miR-105, miR-767, miR-432, and miR-433. We propose that the detected lncRNA expression was timespecific during the gestational and postnatal stages. GO and KEGG analyses of the genes targeted by different miRNAs and lncRNAs revealed that these significantly enriched processes and pathways were consistent with skeletal muscle development over time across all sampled stages. We found four visual lncRNA-gene regulatory networks that can be used to explore the function of lncRNAs in sheep and may be valuable in helping improve muscle growth. This study also describes the function of several lncRNAs that interact with miRNAs to regulate myogenic differentiation.

\section{Introduction}

The first three stages of mammalian muscle development are completed during the embryonic stage, and the number of muscle fibers generally does not change after birth. Postnatal muscle growth is mainly triggered by muscle fiber hypertrophy and increased intermuscular fat (Thomas \& Mathias, 2011). In modern animal husbandry, skeletal muscle is considered the most economically important part of an animal's body. Skeletal muscle yield and quality are determined by the animal's muscle fiber type, metabolism, and physiological characteristics. Recent research has been key to understanding the molecular mechanism of skeletal muscle formation, and the important roles that myogenic regulatory factors (MRFs) and myocyte enhancer factor 2 (MEF2) protein families play in skeletal muscle development (Lang et al., 2007; Snyder et al., 2013). It is now recognized that the myogenic process involves more than just the protein-coding gene signaling pathways. microRNAs (miRNAs) are the most widely studied class of non-coding RNA molecules that 
47 participate in muscle production (Wen et al., 2013), but several recent studies have shown that long non-coding 48 RNAs (lncRNAs) may also play a role in muscle differentiation (Gong et al., 2015; Li et al., 2017). LncRNAs are widely found in mammals; have time-, space-, and tissue-specific expression; and have been shown to contribute to multiple processes, including epigenetic, transcriptional, and post-transcriptional regulation (Mingyang et al., 2015). lncRNA-tiny non-coding RNAs (TncRNAs) found in the porcine fetal trophoblast have been found to be upregulated in embryonic skeletal muscle, and in Tongcheng and Changbai pigs, researchers observed expression level differences in fetal skeletal muscle on the 90th day of pregnancy, suggesting that lncRNAs may have an effect on the embryonic development of porcine skeletal muscle (Ren et al. 2010). Transcriptome sequencing analysis of bovine longissimus dorsi (LD), scapula, intercostal, and gluteal muscles revealed that in bovine myoblasts, the lncRNA lncYYW positively regulated the expression of the growth hormone 1 (GH1) gene and its downstream genes AKT1 and PIK3CD. lncYYW was upregulated during myoblast differentiation, and its overexpression increased the number of cells during the $\mathrm{S}$ phase of the cell cycle (Yue et al., 2017). Transcriptome sequencing of 45-, 60-, and 105-day-old goat fetuses and 3-day-old lamb LD muscle tissue identified 3,981 lncRNAs that were highly conserved in all four stages. A two-timepoint comparison found 577 differentially expressed lncRNAs that may have specific biological effects on early goat muscle development (Zhan et al., 2016). A previous study used strand-specific Ribo-Zero RNA technology to sequence the LD muscles of Hu sheep at three important developmental stages (fetus, lamb, and adult), and obtained a total of 6,924 lncRNAs. The differentially expressed lncRNAs were shown to contribute to biological processes during the embryonic stage, including organ morphogenesis and skeletal and muscle development. This study was the first to systematically analyze lncRNAs in Hu sheep muscles and deliver valuable information on sheep muscle development (Shen et al., 2019). Although progress has been made in identifying and validating specific miRNA and lncRNA targets in skeletal muscle cells, as well as elucidating the functional mechanisms of many miRNAs and lncRNAs in skeletal muscle, the correlations between

To address these gaps in knowledge, this study examined the temporal expression profiles of sheep LD muscle lncRNAs and miRNAs at days 60, 90, and 120 of gestation, as well as at day 0 and 360 following birth. Using RNA sequencing, we were able to discover and add new lncRNAs to the sheep lncRNA and miRNA database. Our results will act as a resource for more thorough insight into the regulatory functions of lncRNAs in sheep, more detailed annotations of the sheep genome, and a better general understanding of mammalian skeletal muscle development. 
81

82

83

84

\section{Materials and Methods}

\section{Ethics statement}

All experimental protocols and procedures were approved by the Institutional Animal Care and Use Committee of Lanzhou Institute of Husbandry and Pharmaceutical Science of the Chinese Academy of Agricultural Sciences (approval no. NKMYD201805, dated 18 October 2018).

\section{Animal and tissue samples}

A total of 15 Gansu Alpine fine wool sheep were used in this study. All Gansu Alpine fine wool sheep were raised in the experimental facilities of Gansu Provincial Sheep Breeding Technology Extension Station (Huangcheng, Gansu, China) under the same conditions with free access to food and water in natural lighting. Caesarean sections were performed on three pregnant ewes from each developmental stage to collect female foetuses at 60, 90, and 120 days of gestation, and six female lambs were collected 0 and 360 days after birth. All animals were slaughtered after being anesthetized with xylazine chlorhydrate. We made all efforts to minimize animal suffering, and the slaughter procedures were carried out in accordance with animal welfare procedures. After slaughter, we collected three gestational (E60, E90, and E120) and two postnatal stage (D0 and D360) LD muscle samples. The tissue samples were snap frozen in liquid nitrogen and stored at $-80^{\circ} \mathrm{C}$ until analysis.

\section{Muscle staining}

We prepared the LD samples for histological sectioning using Carter \& Clarke's (1957) method. The LD samples were first placed in tubes containing $4 \%$ paraformaldehyde solution. After fixation, the samples were embedded, cut into slices, baked, H\&E strained, and mounted (Auber, 1952). We examined the sections using a digital trinocular camera microscope (BA400Digital, McAudi Industrial Co., Ltd., Xiamen, China), and used the image analysis software Motic Images Advanced 3.2 to take and import an image. We then selected the objective lens magnification $(40 \times)$ and the unit of measurement (um), used the polyline tool to measure the data, and exported the measured raw data in the .xls format for sorting and analysis.

\section{RNA isolation, library construction, sequencing, and data analysis}

The total RNA from 15 muscle samples was extracted using TRIzol reagent (Invitrogen, Carlsbad, CA, USA), according to the manufacturer's instructions. We monitored RNA degradation and contamination using 1\% agarose gels. RNA purity and concentration were detected using NanoDrop 2000 (Thermo Fisher Scientific Inc., Waltham, MA, USA), and were further measured using an Agilent 2100 bioanalyzer. Samples with an RNA integrity number (RIN) value greater than 8.0 were used for sequencing. RNA was digested by TruSeq stranded total RNA and a Ribo-Zero Gold Kit (Illumina, San Diego, CA, USA). After the total RNA was extracted, we removed the ribosome RNAs (rRNAs) to retain mRNAs and ncRNAs. The enriched mRNAs and 
113 ncRNAs were then broken down into short fragments using fragmentation buffer and turned into cDNA via 114 reverse transcription and random primers. The second-strand cDNA was synthesized using DNA polymerase I, 115 RNase H, dNTP (dUTP instead of dTTP), and buffer. Next, the cDNA fragments were purified using the 116 Qiaquick PCR extraction kit, end-repaired, combined with poly A, and ligated to Illumina sequencing adapters. 117 We used uracil-N-glycosylase (UNG) to digest second-strand cDNA that had been size-selected by agarose gel 118 electrophoresis, amplified by PCR, and sequenced using an Illumina HiSeq TM 2500 from OE Biotechnology 119 Corporation (Shanghai, China) (Zhou et al., 2016; Zhu et al., 2017). We pooled equal amounts of total RNA 120 from muscle samples across different stages (i.e., E60, E90, E120, D0, and D360; n=3) into one sample. To get 121 high quality clean reads from the sequencing machines, we filtered out those that: 1) contained adapters, 2) had 122 more than $10 \%$ unknown nucleotides $(\mathrm{N})$, or 3 ) contained more than $50 \%$ low quality (Q-value $\leq 20)$ bases.

123 We used the short reads alignment tool Bowtie 2 (Langmead \& Salzberg, 2012a) to map reads to the rRNA 124 database, and then removed the rRNA mapped reads. The remaining reads were used in the transcriptome 125 assembly and analysis. We then mapped the rRNA-removed reads from each sample to the reference genome 126 using TopHat2 (Kim et al., 2013) and the following alignment parameters: 1) a maximum read mismatch of 2 , 127 2) a 50 bp distance between mate-pair reads, and 3) a \pm 80 bp error of distance between mate-pair reads. After aligning them with the reference genome, we re-aligned the unmapped reads (or very poorly mapped reads) using Bowtie2, and split the enriched unmapped reads into smaller groups to find potential splice sites. The section and section positions of these short segments were also predicted. We built a set of splice sites using initial unmapped reads from TopHat2 without relying on known gene annotation (Trapnell et al., 2010). The sequence alignments are not only useful for identifying expressed genes and their quantitative expressions, but

134 Transcript reconstruction was carried out using Cufflinks (Trapnell et al., 2012) which, together with TopHat2, 135 allows biologists to identify new genes and new splice variants of known genes. We preferred to use the program's reference annotation-based transcripts (RABT). Cufflinks constructed faux reads according to the reference gene to compensate for low coverage sequencing. During the last step of assembly, all reassembled fragments were aligned with reference genes and any similar fragments were removed. We used Cuff merge to combine transcripts from different replicas of a group into a comprehensive set of transcripts, and then to merge the transcripts from multiple groups into a comprehensive set of transcripts for further downstream differential expression analysis.

We quantified transcript abundances using RSEM (Li \& Dewey, 2011). Transcript expression levels were normalized using the Fragments Per Kilobase of transcript per Million mapped reads (FPKM) method, and the differentially expressed transcripts of coding RNAs and lncRNAs were individually analysed. To identify differentially expressed transcripts across samples or groups, we used the edge R package (http://www.rproject.org/). We identified transcripts with a fold change $\geq 2$ and a false discovery rate (FDR) $<0.05$ as

148 significant DEGs. DEGs were then subjected to Gene Ontology (GO) function and KEGG pathway enrichment 
149 analysis. We performed gene expression pattern analysis to cluster genes of similar expression patterns from

150 multiple samples (at least three from a specific time point, space, or treatment dose size order). To examine the

151 DEG expression patterns, we normalized the expression data from each sample (in the order of treatment) to 0 ,

$152 \log 2(\mathrm{v} 1 / \mathrm{v} 0), \log 2(\mathrm{v} 2 / \mathrm{v} 0)$, and then clustered them using Short Time-series Expression Miner software (STEM)

153 (Ernst \& Bar-Joseph, 2006). The clustered profiles with p-values $\leq 0.05$ were considered significant, and the

154 DEGs from each profile underwent GO and KEGG pathway enrichment analysis. Using the hypothesis test for 155 p-value calculation and FDR correction (Saldanha, 2004), we defined GO terms or pathways with Q values $\leq$ 1560.05 as significant enriched GO terms or pathways.

\section{Real-time quantitative RT-PCR}

158 The 15 muscle samples were stored at $-80^{\circ} \mathrm{C}$ prior to RNA extraction. We extracted the total RNA from the 15 159 muscle samples using TRIzol reagent (Invitrogen, Carlsbad, CA, USA). RNA purity and concentration were 160 determined using a NanoDrop 2000 spectrophotometer (Thermo Fisher Scientific), and RNA integrity was 161 evaluated using agarose gel electrophoresis staining with ethidium bromide.

Quantification was performed using a two-step reaction process: reverse transcription (RT) and PCR. Each RT reaction consisted of two steps. The first step was to combine $0.5 \mu \mathrm{g}$ of RNA, $2 \mu 1$ of $4 \times \mathrm{g}$ DNA wiper Mix, and $8 \mu$ of nuclease-free H2O. Reactions were performed in a GeneAmp ${ }^{\circledR}$ PCR System 9700 (Applied Biosystems, Foster City, CA, USA) for $2 \mathrm{~min}$ at $42^{\circ} \mathrm{C}$. The second step was to add $2 \mu 1$ of $5 \times$ HiScript II Q RT SuperMix IIa. Reactions were performed in a GeneAmp ${ }^{\circledR}$ PCR System 9700 (Applied Biosystems) for 15 min at $50^{\circ} \mathrm{C}$, then for $5 \mathrm{~s}$ at $85^{\circ} \mathrm{C}$. The $10 \mu \mathrm{RT}$ reaction mix was then diluted $\times 10$ in nuclease-free water and maintained at $-20^{\circ} \mathrm{C}$. Real-time PCR was performed using a LightCycler ${ }^{\circledR} 480$ II Real-time PCR Instrument (Roche, Basel, Switzerland) and $10 \mu \mathrm{l}$ of a PCR reaction mixture that included $1 \mu \mathrm{l}$ of cDNA, $5 \mu \mathrm{l}$ of $2 \times$ ChamQ SYBR qPCR Master Mix, $0.2 \mu \mathrm{l}$ of forward primer, $0.2 \mu \mathrm{l}$ of reverse primer, and $3.6 \mu \mathrm{l}$ of nucleasefree water. Reactions were incubated in a 384 -well optical plate (Roche) at $95^{\circ} \mathrm{C}$ for $30 \mathrm{~s}$, followed by 40 cycles of $10 \mathrm{~s}$ at $95^{\circ} \mathrm{C}$, and $30 \mathrm{~s}$ at $60^{\circ} \mathrm{C}$. Each sample was run in triplicate for analysis. After the PCR cycles, we performed melting curve analysis to validate the specific generation of the expected PCR product. The primer sequences were designed and synthesized by Generay Biotech (Shanghai, China) using the lncRNA and miRNA sequences obtained from the NCBI database (Table S15 and Table S16; Bai et al., 2017). We estimated the PCR efficiency of each gene using standard curve calculation of the four cDNA serial dilution points. Cycle threshold $(\mathrm{Ct})$ values were transformed to quantities using the comparative $\mathrm{Ct}$ method described by Chen et al. (2017). We carried out lncRNA and mRNA data normalization using the GAPDH reference gene, and miRNA data normalization using the U6 reference gene. The lncRNA and miRNA expression levels were normalized (using GAPDH and U6) and estimated using the $2^{-\Delta \Delta \mathrm{Ct}}$ method (Livak \& Schmittgen, 2001). 


\section{Statistical analyses}

183 After comparing the clean reads to the transcription template using Bowtie2 software (Langmead \& Salzberg, 184 2012b), we quantified the transcripts using eXpress software (Roberts \& Pachter, 2013) to obtain FPKM 185 values and mRNA and lncRNA counts. For samples without biological replicates, we calculated the p-value 186 using the Audic_Claverie formula (Tiňo, 2009). miRNAs with p-values $<0.05$ and TMP difference multiples $>$ 1872 were screened.

\section{Results}

\section{Developmental changes in LD muscle across different stages}

190 In this study, we stained the 12 LD in histological sections to analyze the area and circumference of muscle 191 fibers at the various development stages from embryonic to postnatal. Our analysis showed that the area and 192 circumferences of the muscle fibers did not increase over time, but it did decrease during the E90 and E120 periods (Fig. 1A). However, the tightness and evenness of muscle fibers significantly improved as the fetus continued to develop (Fig. 1B). These results suggest that embryonic skeletal muscle growth can be characterized by time.

\section{LncRNA and small RNA sequencing}

To comprehensively analyze sheep lncRNAs and miRNAs across different developmental stages, we constructed five cDNA libraries and five small RNA libraries (E60, E90, E120, D0, and D360) from three LD samples taken at 60, 90, and 120 days of gestation (E60, E90, and E120), and two from the postnatal (D0, D360) developmental stage. During lncRNA sequencing, we generated a total of 504,449,178 raw reads using all five libraries. After discarding adaptor sequences and low-quality reads, we obtained 477,031,266 clean reads (Table S1). Additionally, about $91.87 \%$ (91.70\% - 92.48\%) of the clean reads from each library were mapped to the sheep reference genome (Table S1), confirming the reliability of the sequencing data. During small RNA sequencing, we generated a total of $84,426,880$ raw reads, and we obtained 10,623,459 26,521,572 clean reads (97\%) ranging in size from 18 to $30 \mathrm{nt}$ (Table S2). About 8,513,544 - 24,586,094 reads in the LW samples were perfectly mapped to the reference genome (GCF_000298735.2_Oar_v4.0), amounting to $72.2 \%-92.7 \%$ of the clean reads (Table S2). Our sequencing results showed that most of the small RNA sequences were between 21 and $23 \mathrm{nt}$ in length, which was consistent with the length distribution of the Dicer products and indicated that the sequencing results were good quality and could be used for follow-up analysis.

\section{Differential expression analysis of sheep mRNAs and IncRNAs}

To investigate the key mRNAs and lncRNAs involved in regulating sheep skeletal muscle development, we used RNA-seq datasets from five time points (three gestation stages and two postnatal stages) to characterize their time-specific expression patterns. When comparing the gene expression levels across the five 
214 developmental stages, we found 693 (352 upregulated) DEGs between E60 and E90 (Table S3), 799 (278

215 upregulated) DEGs between E90 and E120 (Table S4), 929 (672 upregulated) DEGs between E120 and D0

216 (Table S5), and 815 (257 upregulated) DEGs between D0 and D360 (Fig. 2A \& B, Table S6). When analyzing

217 these DEGs, we found that 478, 535, 633, and 580 genes were uniquely expressed in one of the two samples in

218 E60 vs. E90, E90 vs. E120, E120 vs. D0, and D0 vs. D360, respectively (Fig. 2A). We detected two of these

219 DEGs, FBN2 (gene ID: 101104991) and HR (gene ID: 443241), across all four comparisons. We also analyzed

220 the differentially expressed lncRNAs (DE-lncRNAs) between E60 vs. E90, E90 vs. E120, E120 vs. D0, and

221 D0 vs. D360, and detected 206, 239, 148, and 101 DE-lncRNAs, respectively (Fig. 2C \& D, Tables S7-S10).

222 We detected six of these DE-lncRNAs, TCONS_00009825, TCONS_00028121, TCONS_00122104,

223 TCONS_00146167, TCONS_00189359, and TCONS_00195408, across all four comparisons. In the

224 embryonic stage, the following gene expressions were significant: TCONS_00054028, TCONS_00023110,

225 and TCONS_00150168 (Fig. 2E). Similarly, we found post-birth differential expression of other related genes,

226 including TCONS_00016124, TCONS_00127579, TCONS_00009472, and TCONS_00014919 (Fig. 2F). Five

227 differentially expressed lncRNAs were selected for qRT-PCR analysis (Table 1), and their differential

228 expression was consistent with the RNA-Seq results. In summary, we found temporal-specific expression of

229 the detected lincRNAs during the gestational and postnatal stages.

\section{GO and KEGG pathway analysis}

231 We used KEGG pathway analysis of DE-lncRNA target genes to identify the pathways that were enriched in

232 DE-lncRNA target genes. When comparing E60 vs. E90, E90 vs. E120, E120 vs. D0, and D0 vs. D360, we

233 found that in the most significantly enriched pathways, DE-lncRNA target genes participated in signal

234 transduction, the endocrine system, the nervous system, cell growth and death folding, sorting and degradation,

235 the immune system, cellular community eukaryotes, translation, amino acid metabolism, and the carbohydrate

236 metabolism pathways (Fig. 3). The top 20 significantly enriched KEGG analyses for each comparison's DE-

237 lncRNAs are shown in Figure S1. GO enrichment analysis of DE-lncRNA targeted genes also delivered a large

238 number of significant annotations, but in order to examine temporal changes in skeletal muscle development,

239 we provided the detailed results from four adjacent DE-lncRNA comparisons. In the E60 vs. E90 comparison,

$240 \mathrm{~T}=$ the top $30 \mathrm{GO}$ terms that were significantly related to genes targeted by total lncRNAs included male

241 meiotic nuclear division, mitotic spindle assembly checkpoint, kinetochore, cell, protein kinase activity, and

242 microtubule plus-end binding (Figure S2). In the E90 vs. E120 comparison, the most significantly enriched

243 GO terms for the total lncRNAs were associated with muscle development, and included signal peptide

244 processing, activation of MAPKK activity, L-type voltage-gated calcium channel complex, voltage-gated

245 calcium channel complex, histone acetyltransferase complex, receptor signaling complex scaffold activity, and

246 Rho GTPase binding (Figure S3). In the E120 vs. D0 comparison, the top 30 processes for down and

247 upregulated lncRNAs were associated with male meiotic nuclear division, cellular response to leukemia

248 inhibitory factor, intercellular bridge, and integral component of endoplasmic reticulum membrane (Figure

Peer] reviewing PDF | (2019:10:42560:4:0:NEW 18 Aug 2020) 
249 S4). In the D0 vs. D360 comparison, we found associations with male meiotic nuclear division, cellular

250 response to leukemia inhibitory factor, microtubule organizing center, and integral component of endoplasmic

251 reticulum membrane (Figure S5). Across the four comparisons, we observed significant changes in skeletal

252 muscle development during the prenatal and neonatal stages.

\section{LncRNA-gene interaction network construction}

254 We extracted the candidate sequences by screening out lncRNAs and genes that were not on the same

255 chromosome as the candidate targets. We used the RNA interaction software RIsearch (Wenzel et al., 2012) to

256 predict the binding of candidate lncRNAs and genes at the nucleic acid level, with the number of bases directly

257 interacting with each other according to the two nucleic acid molecules was no less than 10, and the free

258 energy of base binding was no more than -50 . The possible regulatory interaction networks between lncRNAs

259 and their target genes (mRNAs) were constructed. In the E60 vs. E90 comparison, the lncRNA-gene

260 interaction network was comprised of 15 lncRNAs and 66 protein-coding genes with close networks; among

261 these, TCONS_00196403 and TCONS_00196407 constructed complex network relationships with targeted

262 coding genes (Fig 4A). In the E90 vs. E120 comparison, the lncRNA-gene interaction network was made up of

263 complex network nodes and lncRNA-gene connections between 34 lncRNAs and 79 protein-coding genes.

264 TCONS_00029967 and TCONS_00088616 had a strong correlation with protein-coding genes (Fig 4B). In the

265 E120 vs. D0 comparison, the lncRNA-gene interaction network was comprised of complex network nodes and

266 lncRNA-gene connections between 25 lncRNAs and 95 protein-coding genes. TCONS_00181895,

267 TCONS_00149972, and TCONS_00098734 had a strong correlation with protein-coding genes (Fig 4C). In

268 the D0 vs. D360 comparison, the lncRNA-gene interaction network was comprised of complex network nodes

269 and lncRNA-gene connections between 11 lncRNAs and 86 protein-coding genes. TCONS_00196411,

270 TCONS_00157638, and TCONS_00126396 had a strong correlation with protein-coding genes (Fig 4D).

\section{Temporal-specific expression of the detected sheep miRNAs}

272 The miRNA expression level comparisons across the five developmental stages revealed that there were 328

273 (265 upregulated) DEGs between E60 and E90 (Table S11), 302 (243 upregulated) DEGs between E90 and

274 E120 (Table S12), 295 (166 upregulated) DEGs between E120 and D0 (Table S13), and 184 (110 upregulated)

275 DEGs between D0 and D360 (Fig. 5A, Table S14). We found that the miRNA expression levels during the

276 embryonic and postnatal stages differed significantly. During the embryonic stage, the differentially expressed

277 miRNAs were miR-134, miR-2387, miR-105, miR-3957, miR-493, miR-541, miR-767, miR-432, and miR-433

278 (Fig. 5B). We also conducted a differential analysis of the miRNAs based on their expression levels. The

279 miRNAs with expression levels that changed more than two times and with $\mathrm{P}$ values $<0.05$ were deleted. The

280 results showed that some miRNAs were differentially expressed after birth, specifically miR-22, miR-365, miR-

281 3556, miR-29, miR-193, miR-150, miR-1, and miR-133 (Fig. 5C). Five differentially expressed miRNAs were

282 selected for qRT-PCR analysis (Table 1), and their differential expression was consistent with the RNA-Seq 
283

284

285

286

287

288

289

290

291

292

293

294

295

296

297

298

299

300

301

302

303

304

305

306

307

308

309

310

311

312

313

314

315

316

results. We generated a heatmap based on the expression patterns at all time points and across all known expressed miRNAs (Fig. 5D). The significantly enriched processes and observed pathways were consistent with the temporal changes in skeletal muscle development across all sampled stages.

\section{miRNA target gene prediction and functional analysis}

Most animal miRNAs were not completely complementary to their target mRNA, mainly in the 3 ' non-coding region (3' UTR) of the target mRNA, and their mechanism of action is translation inhibition. Previous studies also found that animal miRNAs could target the $5^{\prime}$ end of the mRNA as well as the coding region (Aleksandra et al., 2013). In this study, we used the miRanda algorithm (John et al., 2004) to predict miRNA target genes. We detected 216, 143, 198, and 204 differentially expressed miRNAs and 33,106, 26,431, 29,049 and 27,554 target genes in the E60 vs. E90, E90 vs. E120, E120 vs. D0, and D0 vs. D360 comparisons, respectively. The high expression of novel 129_mature in E60 vs. E90 indicates that the expression product of the target AHNAK2 gene is related to AHNAK nucleoprotein 2 and transcript variant $\mathrm{X} 1$, and is involved in the nucleus, cytoplasm, cytosol, plasma membrane, $\mathrm{Z}$ disc, T-tubule, cytoplasmic vesicle membrane, and sarcolemma pathways. The high expression of novel 410_mature in E90 vs. E120 suggests that the expression product of the target AHNAK2 gene is mainly related to the S-phase response (cyclin-related). The high expression of novel 360_mature in E120 vs. D0 and D0 vs. D360 shows that the expression product of the target PASD1 gene is related to the PAS domain containing 1 and transcript variant $\mathrm{X} 1$, and is involved in the transcription coactivator binding, nucleus, nuclear speck, negative regulation of circadian rhythm, negative regulation of transcription, DNA-templated, rhythmic process, and Cry-Per complex pathways. We performed GO enrichment analysis at GO Level 2 for all target genes and the genes of differentially expressed miRNAs. The biological processes involved included cellular process, biological regulation, metabolic process, response to stimulus, and regulation of biological process (Fig. S6 \& S7).

\section{Discussion}

In this study, we systematically described the lncRNA and miRNA succession processes during the three prenatal stages and two postnatal stages in sheep embryo LD. We found that lncRNA and miRNA expression were time-specific, and that the differentially expressed miRNAs at the embryonic stage and after birth were also different. miRNAs played a regulatory role during multiple stages of skeletal muscle development, and participated in skeletal muscle stem cell proliferation, differentiation, migration, and resting, myoblast proliferation and differentiation, muscle fiber type conversion, energy metabolism, and other processes (Sun et al., 2010b). For example, we found high expression abundance of miR-1 after birth. Previous studies found that the miR-1's biological function is to promote myogenic differentiation. The main target genes were Hdac4, Pax3, Pax7, Notch3, Hdac2, ND1, and Cox1 (Sun et al., 2010a). In C2C12 cells, miR-1 complements the mitochondrial oxidative phosphorylation-related genes Coxl and ND1 when promoting target gene 
317 translation and upregulating mitochondrial energy metabolism (Zhang et al., 2014). Additionally, we found

318 high expression abundance of miR-432 during the embryo stage. Previous studies found that miR-432 inhibits

319 the PI3K/AKT/mTOR signaling pathway by targeting E2f3 and P55pik genes, while simultaneously inhibiting 320 myoblast proliferation and differentiation (Ma et al., 2017). These results indicate that different miRNAs play

321 important roles in myoblast proliferation and differentiation at different embryonic stages.

322

323

In ruminants, the prenatal stage is crucial for skeletal muscle development because almost all muscle fibers are formed during this period, not after birth (Du et al., 2010). Previous livestock studies have shown that many miRNAs are highly expressed during the prenatal stages and possibly during skeletal myogenesis (Li et al., 2011; Qin et al., 2013). miRNAs regulate myogenic differentiation by directly inhibiting myogenic transcription factors. For example, miR-186 inhibits terminal muscle differentiation by targeting myogenesis, and miR-181 enhances MyoD activity by inhibiting the negative regulator of the myogenic differentiation antigen Hox A11 (Antoniou et al., 2014). Currently, the main myo-miRs are miR-1, miR-133, and miR-206. miR-1 and miR-133 are expressed in both myocardium and skeletal muscle, while miR-206 is expressed only in skeletal muscle (Eva et al., 2007; Sempere et al., 2004). The main functions of miR-206 are to inhibit myoblast proliferation and promote its differentiation. The main functions of miR-133 are to promote myoblast proliferation, inhibit differentiation (Wang, 2013), and contribute to cell fate determination and muscle regeneration. miR-208, miR-499, and miR-486 are also classified as muscle-specific miRNAs. miR-133 targets Runx2, Trps1, and Prdm16, which are responsible for osteoblast, chondrocyte, and fat cell development, respectively (Ying et al., 2012). that miR-133 inhibits cell differentiation in other directions, and is thus conducive to skeletal muscle development (Hang et al., 2013). When comparing E120 vs. D0 and E60 vs. E90, we found significant upregulation of the highly-expressed miRNAs miR-127 and miR-136. miR-127 is mainly expressed in skeletal muscle and has been found to be upregulated during $\mathrm{C} 2 \mathrm{C} 12$ and satellite cell (SC) differentiation (Zhai et al., 2017). Therefore, it is conceivable that miR-127 is involved in muscle development and functional SC postnatal muscle regeneration processes. miR-136 promotes vascular muscle cell proliferation through the erk1/2 pathway by targeting ppp2r2a in atherosclerosis (Zhang \& Chunfeng, 2015). It has also been suggested that by playing a role in regulating vascular myocyte proliferation during early life, miR-136 ensures the normal differentiation and growth of muscle fibers.

In this study, we found that miR-22, miR-365, miR-3556, miR-29, miR-193, miR-150, miR-1, and miR-133 were differentially expressed during the embryonic stage, and that miR-22 expression was highest in skeletal muscle and gradually upregulated during mouse myoblast cell differentiation. miR-22 overexpression repressed $\mathrm{C} 2 \mathrm{C} 12$ myoblast proliferation and promoted myoblast differentiation into myotubes, whereas miR- 
35322 inhibition showed the opposite results (Wang et al., 2018). miR-22 expression in the longissimus tissues of 354 adult pigs was found to be higher than in 33 and 65 day-old pig embryos (Huang et al., 2008), and our study 355 also found higher miR-22 expression in adult sheep than in sheep embryos. Additionally, miR-365 is located on chromosome 16p13.12 and is involved in cell proliferation and apoptosis in many types of cells (Nie et al., 2012). A previous study found that miR-365 significantly inhibits myoblast cell activity and cell growth, suggesting that miR-365 can markedly suppress duck myoblast proliferation (Sun et al., 2019). In this study, we found that miR-365 expression was higher in adult sheep than in sheep embryos, indicating that the gene may be involved in inhibiting the myoblast proliferation. We also observed that oar-miR-410-3p expression was very high during the embryonic stage, which was in agreement with another study that found that miR410-3p overexpression suppressed invasion, migration, and proliferation, downregulated EMT-associated molecule expression, and promoted apoptosis and apoptotic factor expression in rhabdomyosarcoma cells (Zhang et al., 2019). There have been many functional studies on the four lncRNAs, linc-MD1 (Legnini et al., 2014; Yoon et al., 2013), Yam-1 (Li et al., 2018; Simionescu-Bankston \& Kumar, 2016), sirt1 AS (Ming et al., 2016; Wang et al., 2016), and H19 (Dey et al., 2014; Gao et al., 2014), involved in muscle development. These lncRNAs affect myogenic differentiation by interacting with one or more miRNAs. Linc-MD1 is a musclespecific lncRNA that acts as a competitive RNA in mouse and human myoblasts and as a sponge of miR-133 and miR-135, which regulate Maml1 and Mef2c expression. linc-MD1 downregulation inhibits and linc-MD1 overexpression promotes muscle differentiation. H19 is abundantly expressed in embryonic tissues, is inhibited after birth, and is only expressed in skeletal muscle. H19 exon 1 encodes miR-675, which is an miRNA that induces myogenic differentiation expression. In this study, we found that the downregulation of involved miRNAs regulated the progress of muscle development and biological adhesion, and activated myoblasts during differentiation and fusion between day 60 and 90 of gestation. This result was consistent with the muscle cell generation stages found in fetal sheep and goats (Guo et al., 2016; Picard et al., 2002). Intramuscular fat adipogenesis occurs during the late gestational stage in pigs and sheep, and using the GO processes, we found that target genes were enriched between E120 and D0. This suggests that in goats, miRNA is involved in the formation of intramuscular fat during the prenatal stage.

In order to provide new insights into lncRNA and miRNA function during sheep skeletal development, we systematically described the lncRNAs and miRNAs that regulate sheep skeletal myogenesis and examined their LD muscle temporal expression profiles from gestation to the yearling stage. However, our method involved pooling three muscle samples from each stage into one sample to sequence, which had certain flaws. Before selecting this method, we searched the relevant pool-seq literature in detail, and found that: 1) genome sequencing pools of individuals is a cost-effective approach to determining genome-wide allele frequencies in an unbiased manner from a large number of individuals; 2) once the minimum quality criteria have been met, pool -seq-based allele frequency estimates are accurate and reliable; and 3) pool-seq has been successfully applied across a wide range of bulk segregant analyses, evolution and resequencing studies, evolutionary 
389

390

391

392

393

394

395

396

397

398

399

400

401

402

403

404

405

406

407

408

409

410

411

412

413

414

415

416

417

418

419

420

421

422

genome analyses, and time-series data and cancer genomics analyses (Schlötterer et al., 2014). Additionally, we performed real-time quantitative RT-PCR verification of the differential genes obtained by sequencing, which validated the time-specific lncRNA and miRNA expression patterns and the accuracy of the gene expression quantification.

\section{Conclusion}

In this study, we systematically identified the lncRNAs and miRNAs that regulate sheep skeletal myogenesis, and examined their LD temporal expression profiles from the gestational to postnatal stages. We described a set of lncRNAs, miRNAs, and genes related to LD muscle growth across five developmental stages. Additionally, we provided four visual lncRNA-gene regulatory networks that can be used to further explore lncRNA function in sheep. We also described several lncRNAs that interact with miRNAs to regulate myogenic differentiation. Our results are valuable resources for future studies on lncRNA and miRNA biology, particularly those regarding sheep muscle, and are helpful in understanding lncRNA and miRNA function in sheep. Integrating published data on lncRNAs and miRNAs and their influence on skeletal muscle development in goats and sheep is a critical step towards building a database.

\section{Availability of data and materials}

The dataset supporting the conclusions of this article is available in the NCBI Sequence Read Archive (SRA) repository (SRP188484).

\section{Acknowledgements}

We would like to thank Fanwen Li, Jigang Liu, Yazhou Wen, and Tianzhao Luo from the Gansu Provincial Sheep Breeding Technology Extension Station for their assistance in sample collection.

\section{References}

Aleksandra H, Grzegorz K, Tatiana D, and David T. 2013. Mapping the human miRNA interactome by CLASH reveals frequent noncanonical binding. Cell 153:654-665.

Antoniou A, Mastroyiannopoulos NP, Uney JB, and Phylactou LA. 2014. miR-186 inhibits muscle cell differentiation through myogenin regulation. J Biol Chem 289:3923-3935.

Auber L. 1952. VII.-The Anatomy of Follicles Producing Wool-Fibres, with special reference to Keratinization. Earth and Environmental Science Transactions of The Royal Society of Edinburgh 62:191-254.

Bai R, Yang Q, Xi R, Li L, Shi D, and Chen K. 2017. miR-941 as a promising biomarker for acute coronary syndrome. BMC cardiovascular disorders 17:227.

Carter H, and Clarke W. 1957. Hair follicle group and skin follicle population of Australian Merino sheep. Australian Journal of Agricultural Research 8:91-108. 
423

424

425

426

427

428

429

430

431

432

433

434

435

436

437

438

439

440

441

442

443

444

445

446

447

448

449

450

451

452

453

454

455

456

457

458

459

460

461

462

Chen X, Ma C, Chen C, Lu Q, Shi W, Liu Z, Wang H, and Guo H. 2017. Integration of lncRNA-miRNAmRNA reveals novel insights into oviposition regulation in honey bees. PeerJ 5:e3881.

Dey BK, Pfeifer K, and Dutta A. 2014. The H19 long noncoding RNA gives rise to microRNAs miR$675-3 p$ and miR-675-5p to promote skeletal muscle differentiation and regeneration. Genes \& development 28:491-501.

Du M, Yan X, Tong JF, Zhao J, and Zhu MJ. 2010. Maternal Obesity, Inflammation, and Fetal Skeletal Muscle Development. Biology of Reproduction 82:4-12.

Eva VR, Sutherland LB, Xiaoxia Q, Richardson JA, Joseph H, and Olson EN. 2007. Control of stressdependent cardiac growth and gene expression by a microRNA. Science 316:575-579.

Gao Y, Wu F, Zhou J, Yan L, Jurczak MJ, Lee HY, Yang L, Mueller M, Zhou XB, Dandolo L, Szendroedi J, Roden M, Flannery C, Taylor H, Carmichael GG, Shulman GI, and Huang Y. 2014. The H19/let7 double-negative feedback loop contributes to glucose metabolism in muscle cells. Nucleic Acids Res 42:13799-13811.

Gong C, Li Z, Ramanujan K, Clay I, Zhang Y, Lemire-Brachat S, and Glass D. 2015. A Long Non-coding RNA, LncMyoD, Regulates Skeletal Muscle Differentiation by Blocking IMP2-Mediated mRNA Translation. Developmental Cell 34:181-191.

Guo J, Zhao W, Zhan S, Li L, Zhong T, Wang L, Dong Y, and Zhang H. 2016. Identification and expression profiling of miRNAome in goat longissimus dorsi muscle from prenatal stages to a neonatal stage. PLoS One 11:e165764.

Hang Y, Alessandra P, Soleimani VD, C Florian B, Ghadi A, Stephanie T, Patrick S, Pasan F, Wilfred VI, and Frank G. 2013. MicroRNA-133 controls brown adipose determination in skeletal muscle satellite cells by targeting Prdm16. Cell Metab 17:210-224.

Huang T, Zhu M, Li X, and Zhao S. 2008. Discovery of porcine microRNAs and profiling from skeletal muscle tissues during development. PLoS One 3:e3225.

John B, Enright AJ, Aravin A, Tuschl T, Sander C, and Marks DS. 2004. Human MicroRNA targets. PLoS Biol. 2:e363

Kim D, Pertea G, Trapnell C, Pimentel H, Kelley R, and Salzberg SL. 2013. TopHat2: accurate alignment of transcriptomes in the presence of insertions, deletions and gene fusions. Genome Biol 14:R36.

Lang D, Powell SK, Plummer RS, Young KP, and Ruggeri BA. 2007. PAX genes: Roles in development, pathophysiology, and cancer. Biochemical Pharmacology 73:1-14.

Langmead B, and Salzberg SL. 2012. Fast gapped-read alignment with Bowtie 2. Nature methods 9:357359.

Legnini I, Morlando M, Mangiavacchi A, Fatica A, and Bozzoni I. 2014. A feedforward regulatory loop between HuR and the long noncoding RNA linc-MD1 controls early phases of myogenesis. Molecular cell 53:506-514.

Li B, and Dewey CN. 2011. RSEM: accurate transcript quantification from RNA-Seq data with or without a reference genome. BMC bioinformatics 12:323.

Li T, Wu R, Yong Z, and Zhu D. 2011. A systematic analysis of the skeletal muscle miRNA transcriptome of chicken varieties with divergent skeletal muscle growth identifies novel miRNAs and differentially expressed miRNAs. BMC Genomics 12:1-20.

Peer) reviewing PDF | (2019:10:42560:4:0:NEW 18 Aug 2020) 
463

464

465

466

467

468

469

470

471

472

473

474

475

476

477

478

479

480

481

482

483

484

485

486

487

488

489

490

491

492

493

494

495

496

497

498

499

500

501

Li Y, Chen X, Sun H, and Wang H. 2017. Long non-coding RNAs in the regulation of skeletal myogenesis and muscle diseases. Cancer Letters 417:58-64.

Livak KJ, and Schmittgen TD. 2001. Analysis of relative gene expression data using real-time quantitative PCR and the 2- $\Delta \Delta C$ T method. Methods 25:402-408.

Ma M, Wang X, Chen X, Cai R, and Pang W. 2017. MicroRNA-432 targeting E2F3 and P55PIK inhibits myogenesis through PI3K/AKT/mTOR signaling pathway. RNA Biology 14:347-360.

Ming G, Wu K, Hu K, Chen Y, and Xiao J. 2016. NAMPT regulates senescence, proliferation, and migration of endothelial progenitor cells through the SIRT1 AS IncRNA/miR-22/SIRT1 pathway. Biochemical and biophysical research communications 478:1382-1388.

Quan, M., Chen, J., \& Zhang, D. 2015. Exploring the Secrets of Long Noncoding RNAs. International Journal of Molecular Sciences, 16:5467-5496.

Nie J, Liu L, Zheng W, Chen L, Wu X, Xu Y, Du X, and Han W. 2012. microRNA-365, down-regulated in colon cancer, inhibits cell cycle progression and promotes apoptosis of colon cancer cells by probably targeting Cyclin D1 and Bcl-2. Carcinogenesis 33:220-225.

Picard B, Lefaucheur L, Berri C, and Duclos MJ. 2002. Muscle fibre ontogenesis in farm animal species. Reprod Nutr Dev 42:415-431.

Qin L, Chen Y, Liu X, Ye S, Yu K, Huang Z, Yu J, Zhou X, Chen H, and Mo D. 2013. Integrative Analysis of Porcine microRNAome during Skeletal Muscle Development. PLoS One 8:e72418.

Ren H, Li Y, Tang Z, Yang S, Mu Y, Cui W, Ao H, Du L, Wang L, and Li K. 2010. Genomic structure, chromosomal localization and expression profile of a porcine long non-coding RNA isolated from long SAGE libraries. Anim Genet 40:499-508.

Roberts A, and Pachter L. 2013. Streaming fragment assignment for real-time analysis of sequencing experiments. Nat Methods 10:71.

Saldanha AJ. 2004. Java Treeview-extensible visualization of microarray data. Bioinformatics 20:32463248.

Schlötterer C, Tobler R, Kofler R, and Nolte V. 2014. Sequencing pools of individuals-mining genomewide polymorphism data without big funding. Nature Reviews Genetics 15:749-763.

Sempere LF, Freemantle S, Pitha-Rowe I, Moss E, Dmitrovsky E, and Ambros V. 2004. Expression profiling of mammalian microRNAs uncovers a subset of brain-expressed microRNAs with possible roles in murine and human neuronal differentiation. Genome Biol 5:1-11.

Shen T, Liu Y, Shang J, Xie Q, Li J, Yan M, Xu J, Niu J, Liu J, Watkins PB, Aithal GP, Andrade RJ, Dou X, Yao L, Lv F, Wang Q, Li Y, Zhou X, Zhang Y, Zong P, Wan B, Zou Z, Yang D, Nie Y, Li D, Wang Y, Han X, Zhuang H, Mao Y, Chen C. 2019. Incidence and Etiology of Drug-Induced Liver Injury in Mainland China. Gastroenterology 156:2230-2241.e11.

Simionescu-Bankston A, and Kumar A. 2016. Noncoding RNAs in the regulation of skeletal muscle biology in health and disease. Journal of Molecular Medicine 94:853-866.

Snyder CM, Rice AL, Estrella NL, Aaron H, Kandarian SC, and Naya FJ. 2013. MEF2A regulates the Gtl2Dio3 microRNA mega-cluster to modulate WNT signaling in skeletal muscle regeneration. Development 140:31-42.

Peer] reviewing PDF | (2019:10:42560:4:0:NEW 18 Aug 2020) 
502

503

504

505

506

507

508

509

510

511

512

513

514

515

516

517

518

519

520

521

522

523

524

525

526

527

528

529

530

531

532

533

534

535

536

537

538

539

540

541

Sun W, Hu S, Hu J, Yang S, Hu B, Qiu J, Gan X, Liu H, Li L, and Wang J. 2019. miR-365 inhibits duck myoblast proliferation by targeting IGF-I via PI3K/Akt pathway. Bioscience reports 39:BSR20190295.

Sun Y, Ye J, Drnevich J, Zhao Y, Band M, and Chen J. 2010. Mammalian target of rapamycin regulates miRNA-1 and follistatin in skeletal myogenesis. J Cell Biol 189:1157-1169

Thomas B, and Mathias G. 2011. Transcriptional mechanisms regulating skeletal muscle differentiation, growth and homeostasis. Nature Reviews Molecular Cell Biology 12:349-361.

Tiňo P. 2009. Basic properties and information theory of Audic-Claverie statistic for analyzing cDNA arrays. BMC bioinformatics 10:310.

Trapnell C, Roberts A, Goff L, Pertea G, Kim D, Kelley DR, Pimentel H, Salzberg SL, Rinn JL, and Pachter L. 2012. Differential gene and transcript expression analysis of RNA-seq experiments with TopHat and Cufflinks. Nature protocols 7:562-578.

Trapnell C, Williams BA, Pertea G, Mortazavi A, Kwan G, Van Baren MJ, Salzberg SL, Wold BJ, and Pachter L. 2010. Transcript assembly and quantification by RNA-Seq reveals unannotated transcripts and isoform switching during cell differentiation. Nat Biotechnol 28:511-515.

Wang G, Wang Y, Xiong Y, Chen X, Ma M, Cai R, Gao Y, Sun Y, Yang G, and Pang W. 2016. Sirt1 AS IncRNA interacts with its mRNA to inhibit muscle formation by attenuating function of miR-34a. Sci Rep 6:21865.

Wang H, Zhang Q, Wang B, Wu W, Wei J, Li P, and Huang R. 2018. miR-22 regulates C2C12 myoblast proliferation and differentiation by targeting TGFBR1. European journal of cell biology 97:257268.

Wang X. 2013. MicroRNA in myogenesis and muscle atrophy. Current Opinion in Clinical Nutrition \& Metabolic Care 16:258-266.

Wen L, Nie Q, and Zhang X. 2013. MicroRNAs Involved in Skeletal Muscle Differentiation. Journal of Genetics \& Genomics 40:107-116.

Ying Z, Rong-Lin X, Jonathan G, Kimberly LB, Stein JL, Lian JB, Wijnen AJ, Van, and Stein GS. 2012. Control of mesenchymal lineage progression by microRNAs targeting skeletal gene regulators Trps1 and Runx2. Journal of Biological Chemistry 287:21926-21935.

Yoon J, Abdelmohsen K, and Gorospe M. 2013. Posttranscriptional gene regulation by long noncoding RNA. Journal of molecular biology 425:3723-3730.

Yue Y, Jin C, Chen M, Zhang L, Liu X, Ma W, and Guo H. 2017. A lncRNA promotes myoblast proliferation by up-regulating GH1. In Vitro Cellular \& Developmental Biology Animal 53:1-7.

Zhai L, Wu R, Han W, Zhang Y, and Zhu D. 2017. miR-127 enhances myogenic cell differentiation by targeting S1PR3. Cell Death \& Disease 8:e2707.

Zhan S, Dong Y, Zhao W, Guo J, Zhong T, Wang L, Li L, and Zhang H. 2016. Genome-wide identification and characterization of long non-coding RNAs in developmental skeletal muscle of fetal goat. $B M C$ Genomics 17:666.

Zhang X, Zuo X, Yang B, Li Z, Xue Y, Zhou Y, Huang J, Zhao X, Zhou J, Yan Y, Zhang H, Guo P, Sun H, Guo L, Zhang Y, and Fu XD. 2014. MicroRNA Directly Enhances Mitochondrial Translation during Muscle Differentiation. Cell 158:607-619. 
542 543
Zhang CF, Kang K, Li XM, and Xie BD. 2015. MicroRNA-136 Promotes Vascular Muscle Cell Proliferation Through the ERK1/2 Pathway by Targeting PPP2R2A in Atherosclerosis. Current Vascular Pharmacology 13:405-412.

Zhang L, Pang Y, Cui X, Jia W, Cui W, Liu Y, Liu C, and Li F. 2019. MicroRNA-410-3p upregulation suppresses proliferation, invasion and migration, and promotes apoptosis in rhabdomyosarcoma cells. Oncology letters 18:936-943.

Zhou Y, Wu J, Geng P, Kui X, Xie Y, Zhang L, Liu Y, Yin N, Zhang G, and Yi S. 2016. MicroRNA profile analysis of host cells before and after wild human rotavirus infection. Journal of medical virology 88:1497-1510.

Zhu B, Xu M, Shi H, Gao X, and Liang P. 2017. Genome-wide identification of lncRNAs associated with chlorantraniliprole resistance in diamondback moth Plutella xylostella (L.). BMC Genomics $18: 380$. 
555

556

557

558

559

560

561

562

563

564

565

566

567

568

569

570

571

572

573

574

575

576

577

578

579

580

581

582

583

584

\section{Figure legends:}

Figure 1. HE-stained longissimus dorsi tissue section.

(A) The trend chart of the muscle fibre area and circumference. (B) Comparison of the longissimus muscle of sheep foetuses at different developmental stages by H.E. staining. The 12 sheep LD were stained in histological sections to analyse the muscle fibre area and circumference at various stages. Statistics of measurements were analysed by one-way ANOVA with a Tukey's test, * represents $\mathrm{P}<0.05$, ** represents $\mathrm{p}$ $<0.01$.

\section{Figure 2. Number of DEGs and DE-IncRNAs at different time stages.}

(A) The number of DEGs across four comparisons: E60 vs. E90, E90 vs. E120, E120 vs. D0, and D0 vs. D360. (B) The number of up and downregulated DEGs across four comparisons: E60 vs. E90, E90 vs. E120, E120 vs. D0, and D0 vs. D360. (C) The number of DE-lncRNAs across four comparisons: E60 vs. E90, E90 vs. E120, E120 vs. D0, and D0 vs. D360. (D) The number of up and downregulated DE-lncRNAs across the four comparisons: E60 vs. E90, E90 vs. E120, E120 vs. D0, and D0 vs. D360. The differentially expressed heatmap of significant lncRNA down (E) and upregulation (F).

Figure 3. The top KEGG enrichment analyses of the differentially expressed lncRNAs in E60 vs. E90 (A), E90 vs. E120 (B), E120 vs. D0 (C), and D0 vs. D360 comparisons (D).

Figure 4. The lncRNA-gene network for the comparisons of E60 vs. E90 (A), E90 vs. E120 (B), E120 vs. D0 (C), and D0 vs. D360 (D).

\section{Figure 5. Sheep LD muscle miRNA features.}

(A) The number of up and downregulated miRNAs across the four comparisons: E60 vs. E90, E90 vs. E120, E120 vs. D0, and D0 vs. D360. The differentially expressed heatmap of significant miRNA down (B) and upregulation (C). (D) A heatmap of miRNAs across five time stages. Each row represents the expression levels of all detected miRNAs.

\section{Table legends:}

Table 1. Validation of RNA-seq results by quantitative RT-PCR. QPCR indicates the gene expression level calculated by the $2^{-\Delta \Delta \mathrm{Ct}}$ method and quantitative RT-PCR, and FPKM indicates the gene expression level calculated by sequencing. 


\section{Supplemental Tables}

586 Table S1. LncRNA sequence statistics for the five samples.

587 Table S2. Small RNA sequence statistics for the five samples.

588 Table S3. Differentially expressed mRNAs in the E60 and E90 comparisons.

589 Table S4. Differentially expressed mRNAs in the E90 and E120 comparisons.

590 Table S5. Differentially expressed mRNAs in the E120 and D0 comparisons.

591 Table S6. Differentially expressed mRNAs in the D0 and D360 comparisons.

592 Table S7. Differentially expressed lncRNAs and their corresponding target genes in the E60 and E90

593 comparisons.

594 Table S8. Differentially expressed lncRNAs and their corresponding target genes in the E90 and E120 595 comparisons.

596 Table S9. Differentially expressed lncRNAs and their corresponding target genes in the E120 and D0 597 comparisons.

598 Table S10. Differentially expressed lncRNAs and their corresponding target genes in the D0 and D360 599 comparisons.

600 Table S11. Differentially expressed miRNAs and their sequence in the E60 and E90 comparisons.

601 Table S12. Differentially expressed miRNAs and their sequence in the E90 and E120 comparisons.

602 Table S13. Differentially expressed miRNAs and their sequence in the E120 and D0 comparisons.

603 Table S14. Differentially expressed miRNAs and their sequence in the D0 and D360 comparisons.

604 Table S15. LncRNA primers for qRT-PCR.

605 Table S16. miRNA primers for qRT-PCR.

606

607

608 Supplemental Figures

609 Figure S1. The top 20 KEGG enrichment analyses of total differentially expressed lncRNAs in the E60 and 610 E90 (A), E90 and E120 (B), E120 and D0 (C), and D0 and D360 (D) comparisons.

611 Figure S2. GO annotation for predicted target genes of differentially expressed lncRNAs in the E60 and E90 612 comparisons. The top 30 significantly enriched GO terms for the predicted target genes are shown.

613 Figure S3. GO annotation for predicted target genes of differentially expressed lncRNAs in the E90 and E120 614 comparisons. The top 30 significantly enriched GO terms for the predicted target genes are shown.

615 Figure S4. GO annotation for predicted target genes of differentially expressed lncRNAs in the E120 and D0 616 comparisons. The top 30 significantly enriched GO terms for the predicted target genes are shown.

617 Figure S5. GO annotation for predicted target genes of differentially expressed lncRNAs in the D0 and D360 618 comparisons. The top 30 significantly enriched GO terms for the predicted target genes are shown. 
619 Figure S6. Comparison of E60 vs. E90 and E90 vs. E120 miRNA target genes and all genes at GO Level 2 620 distribution.

621 Figure S7. Comparison of E120 vs. D0 and D0 vs. D360 miRNA target genes and all genes at GO Level 2 622 distribution.

623

624

625 
Figure 1

HE stained longissimus dorsi tissue section
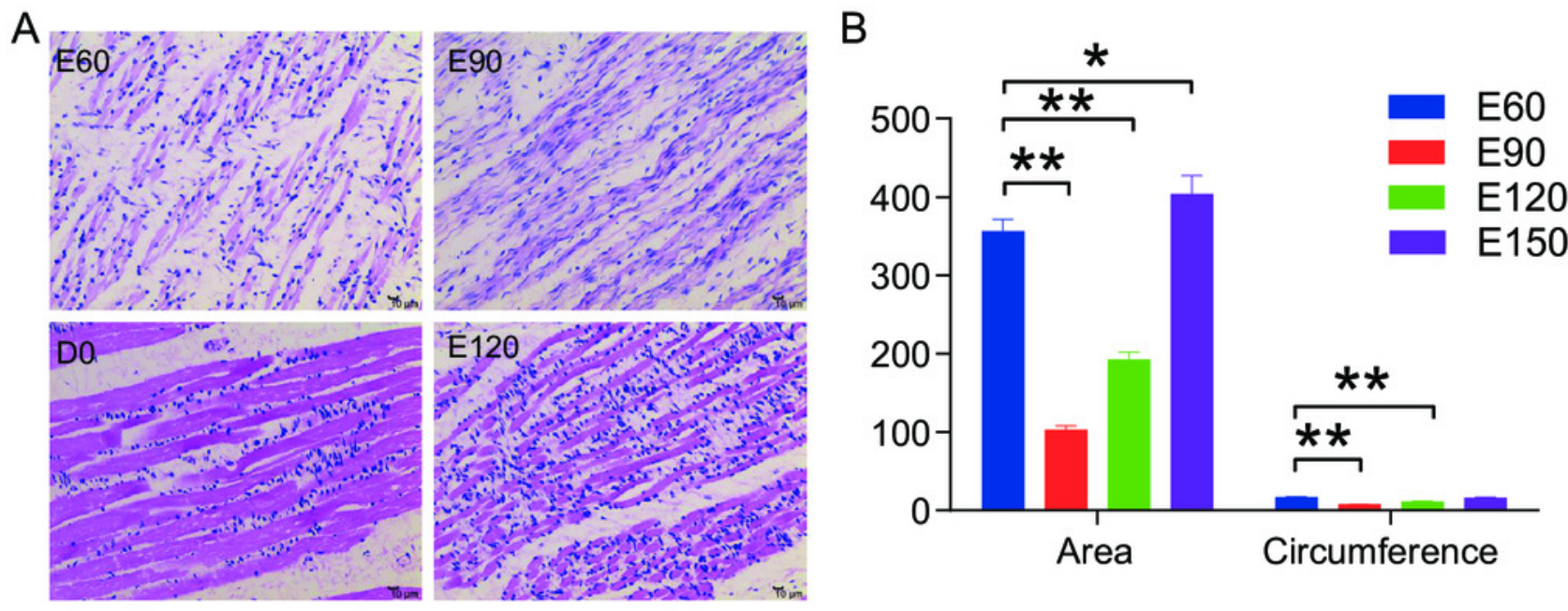
Figure 2

Number of DEG and DE-IncRNAs at different time stage comparisons. 
A
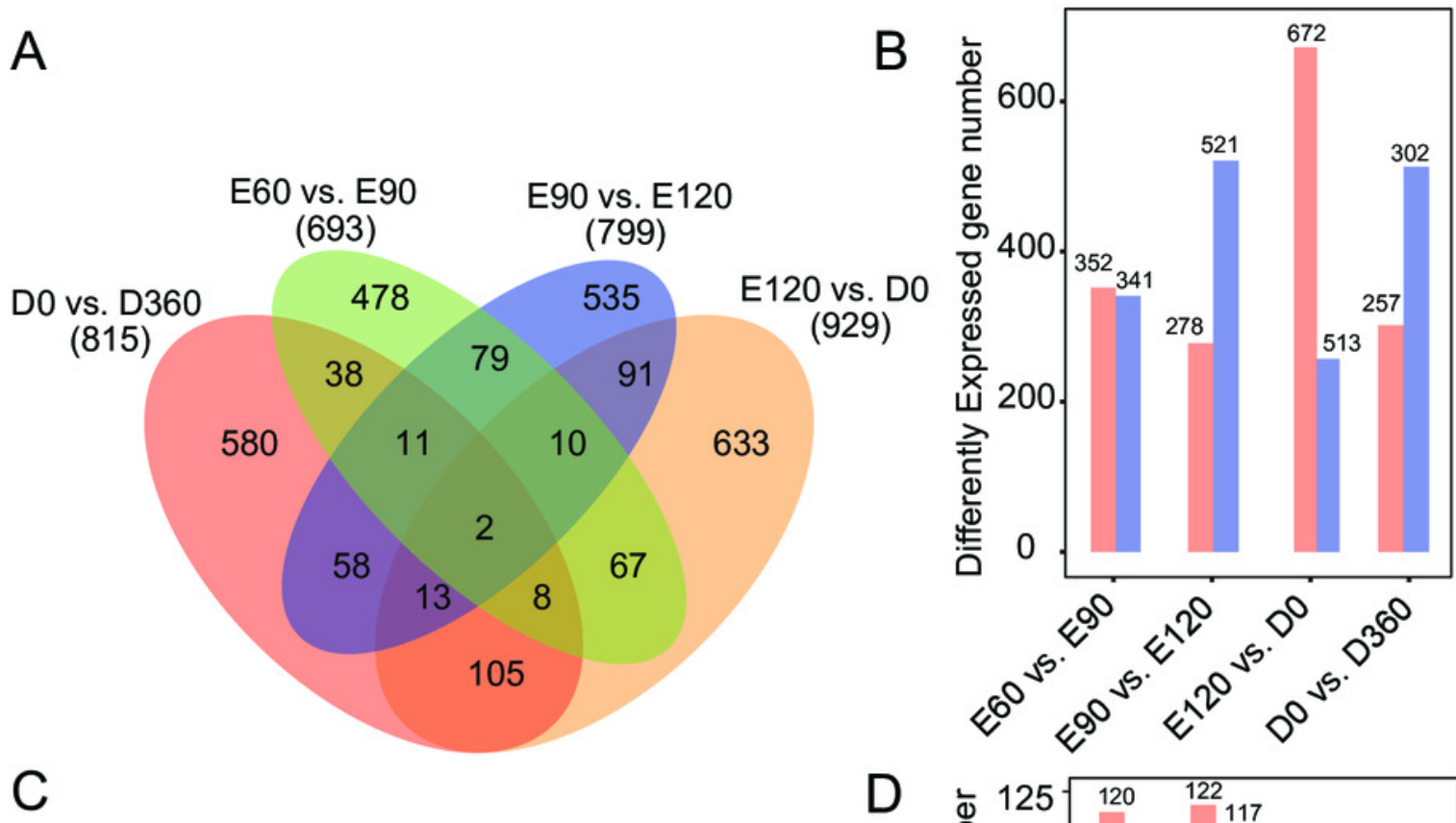

Type

Up

Down

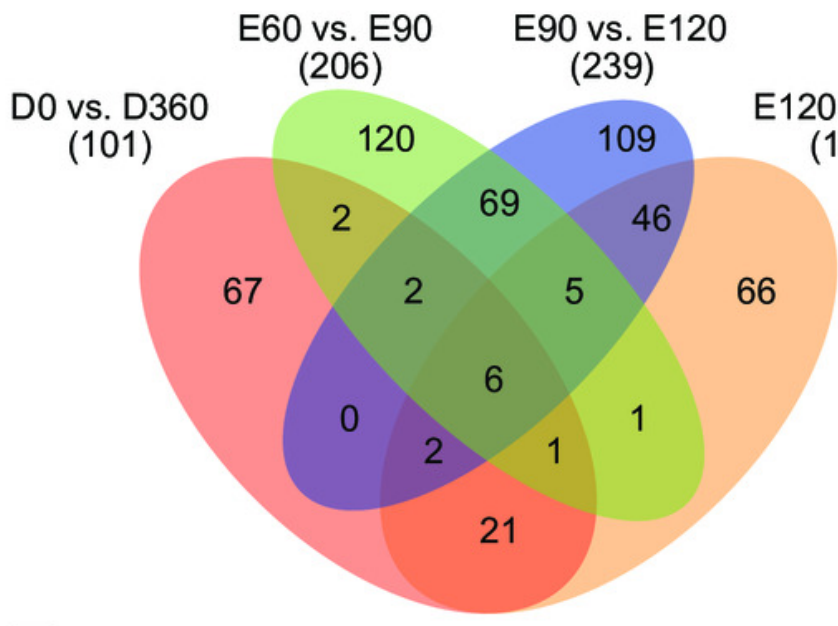

E

(148)
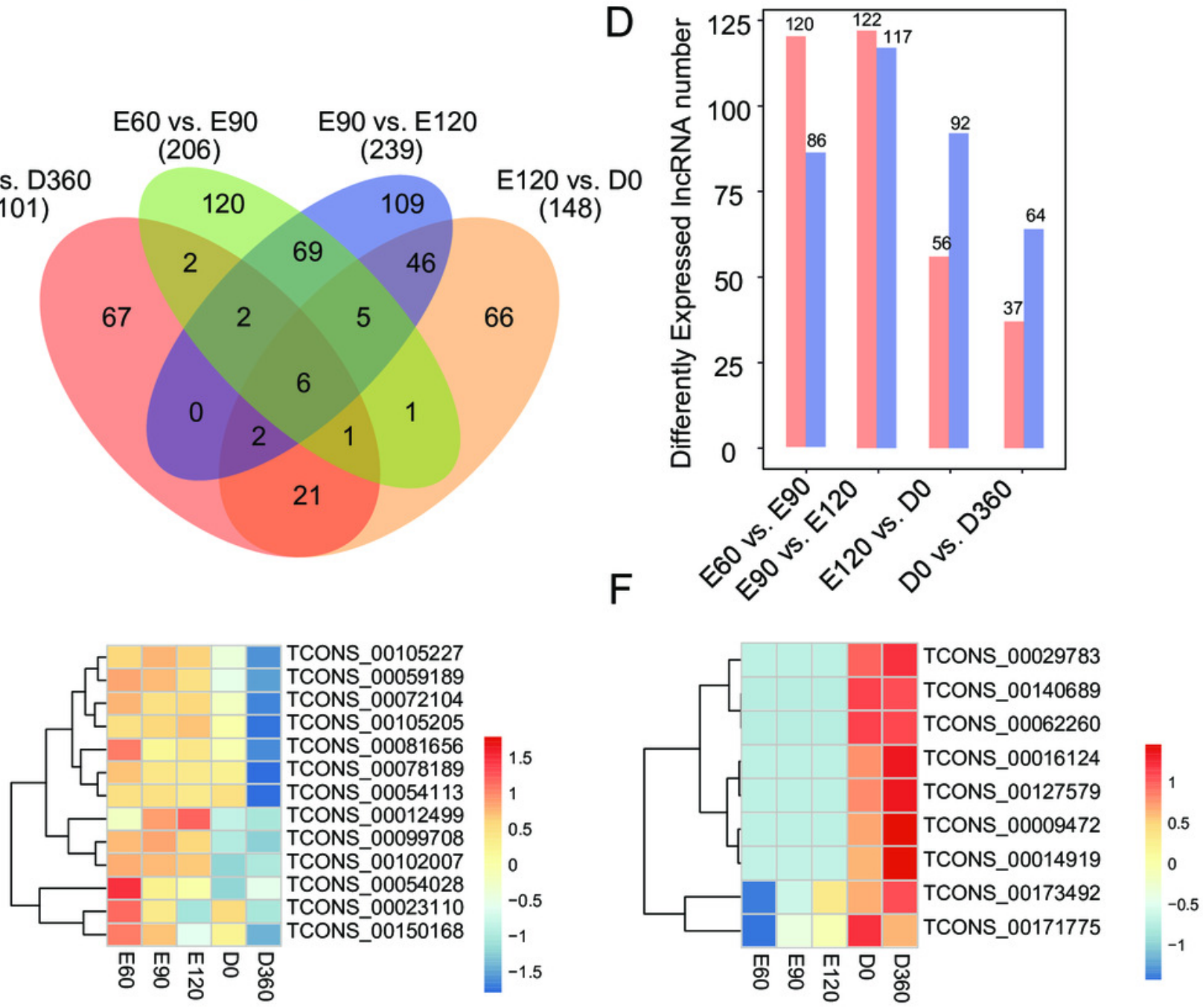
Figure 3

The top KEGG enrichment analyses of the differentially expressed IncRNAs in E60 vs. E90, E90 vs. E120, E120 vs. D0, and D0 vs. D360 comparisons.
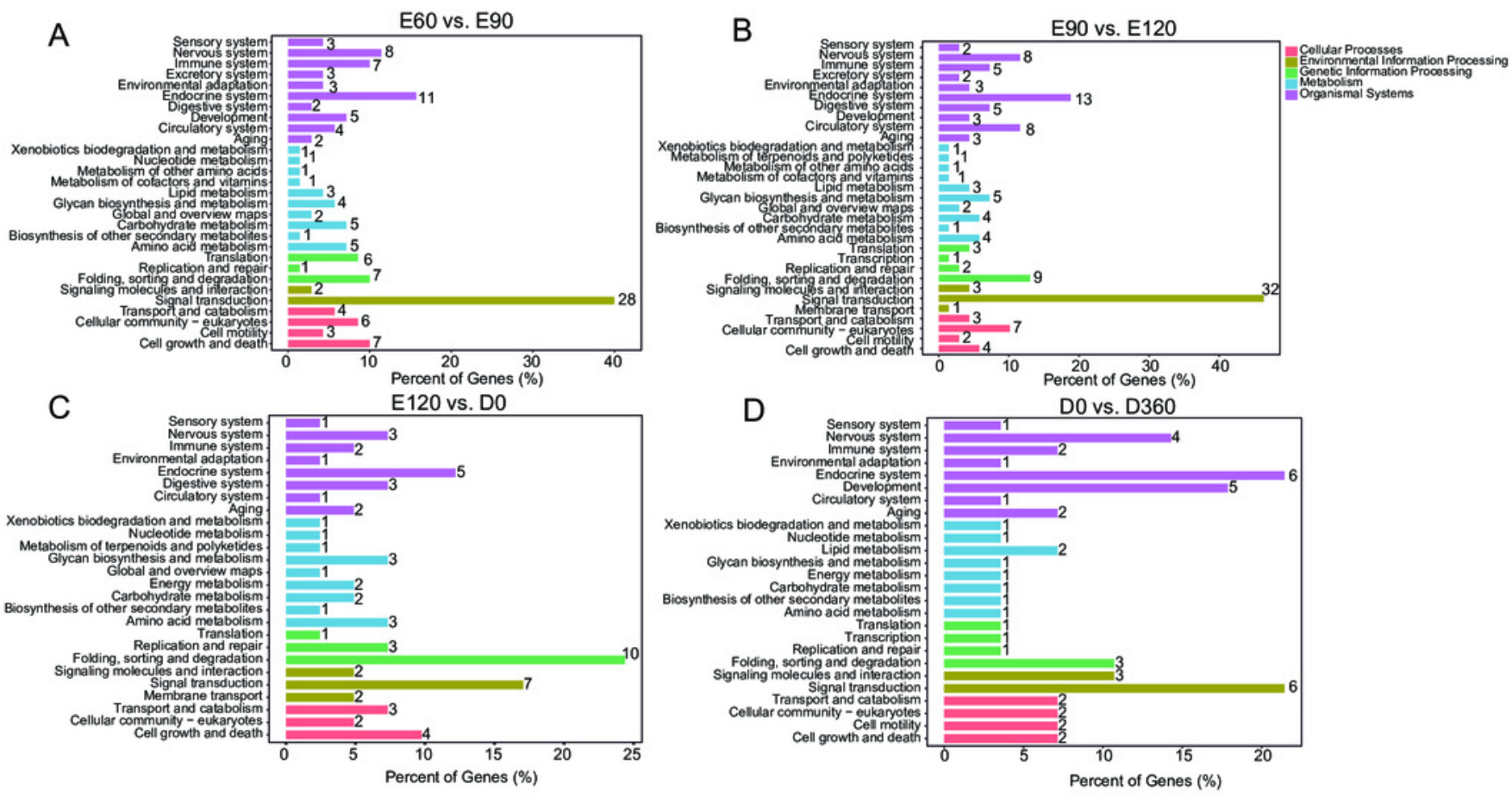
Figure 4

LncRNA-gene network for the comparisons of E60 vs. E90, E90 vs. E120, E120 vs. D0 and D0 vs. D360.

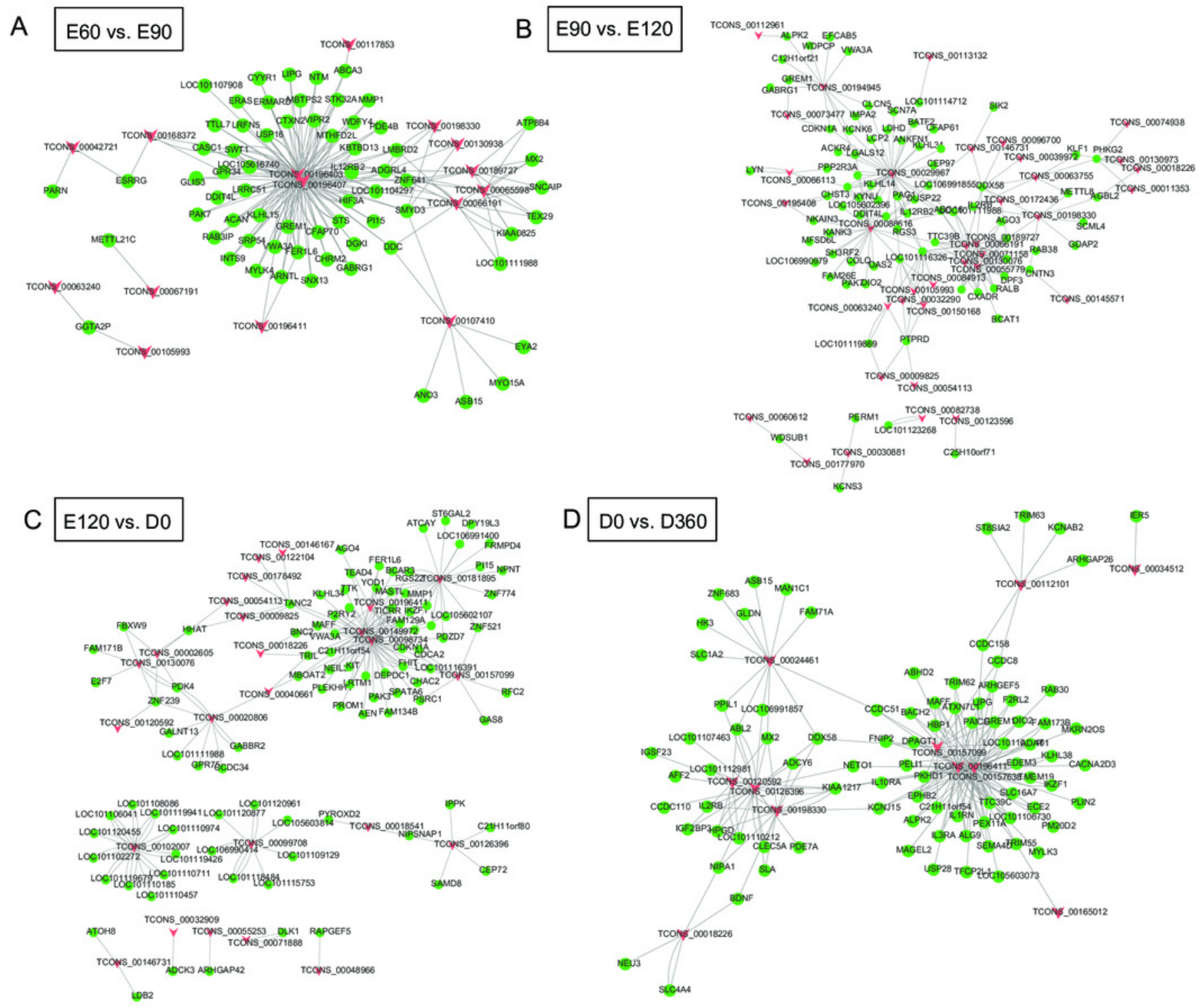


Figure 5

The features of sheep longissimus dorsi muscle miRNAs.

A

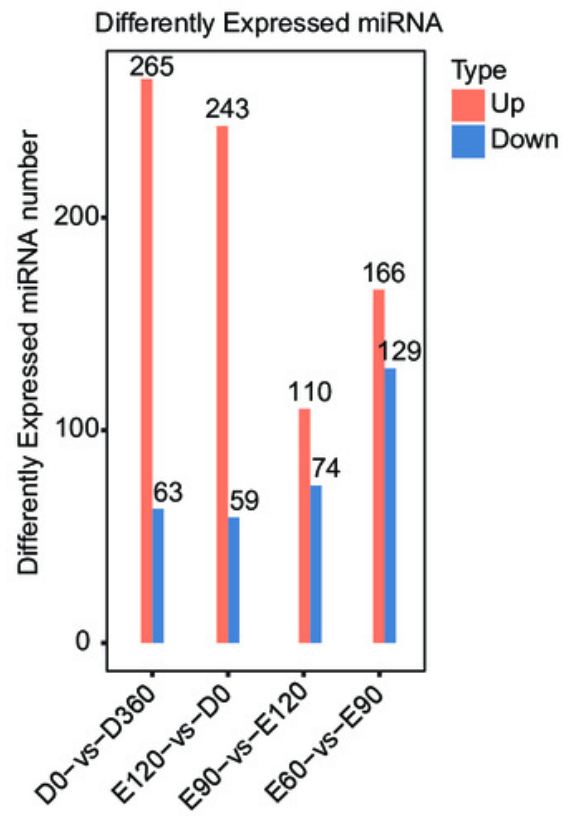

C

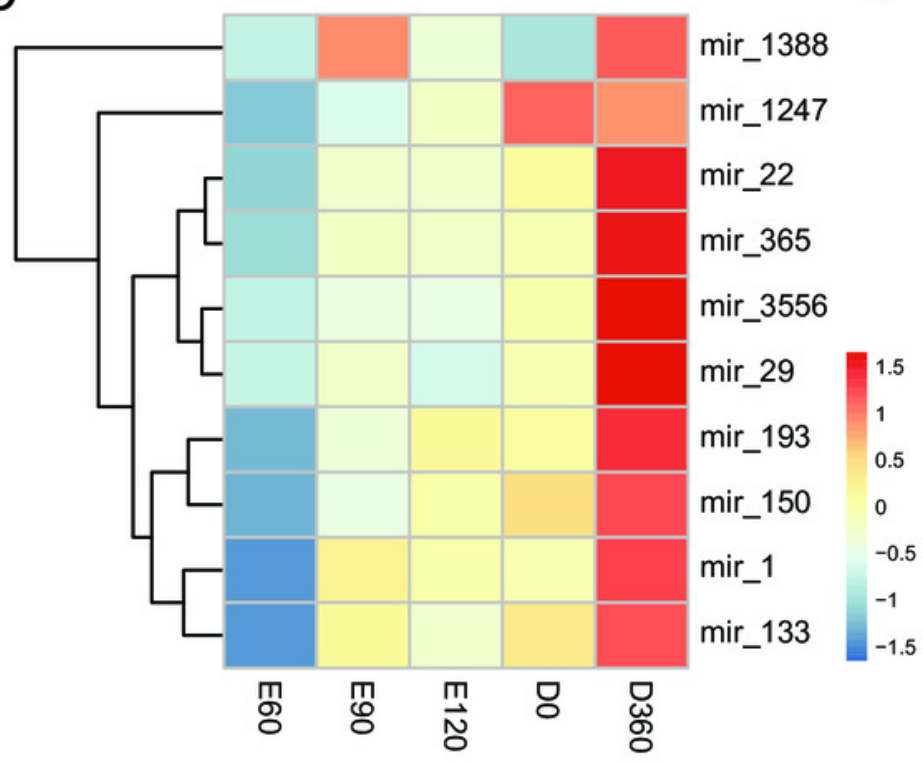

B

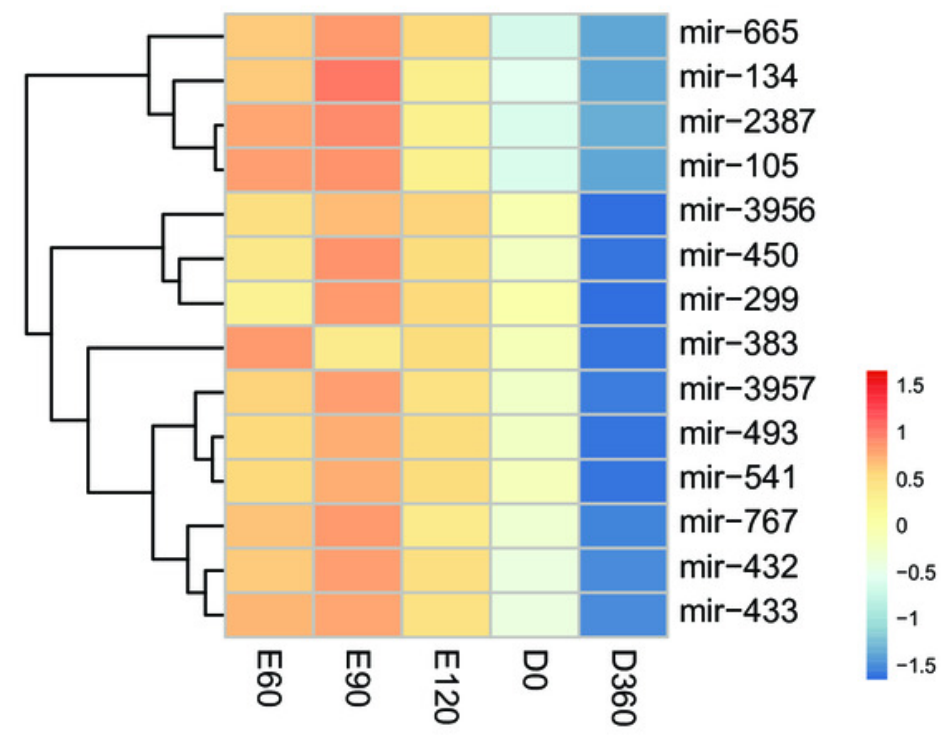

D

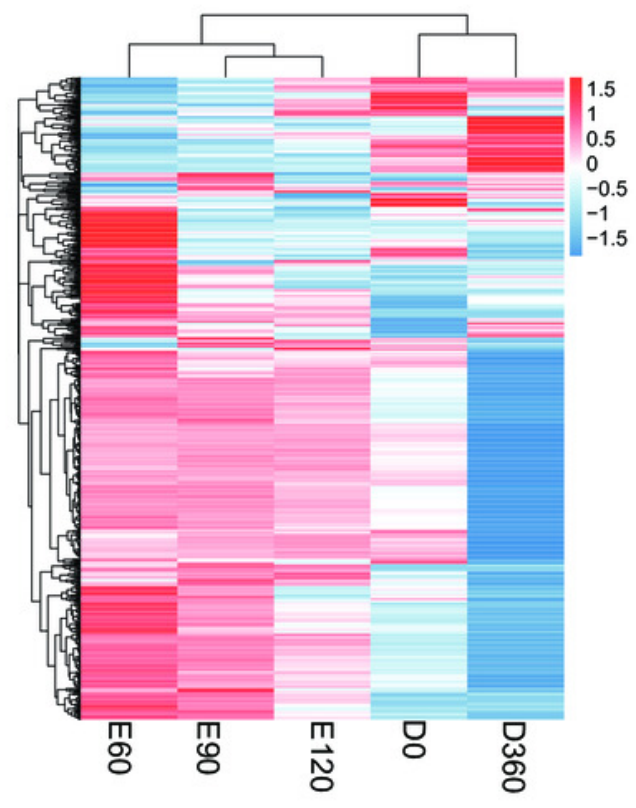




\section{Table $\mathbf{1}$ (on next page)}

Validation of RNA-seq results by using quantitative RT-PCR. QPCR indicates the expression level of the gene calculated by the $2^{-\Delta \Delta C t}$ method using quantitative RT-PCR, FPKM indicates the gene expression level calculated by sequencing. 
1 Table 1. Validation of RNA-seq results by using quantitative RT-PCR. QPCR indicates the expression level of 2 the gene calculated by the $2^{-\Delta \Delta C t}$ method using quantitative RT-PCR, FPKM indicates the gene expression level 3 calculated by sequencing.

\begin{tabular}{|c|c|c|c|c|c|c|c|}
\hline Item & Accession No. & Genes & E60 & E90 & E120 & D0 & D360 \\
\hline \multirow{10}{*}{ miRNA } & \multirow{2}{*}{ oar-miR-103 } & QPCR & 1.79 & 2.35 & 1.86 & 1.00 & 1.29 \\
\hline & & FPKM & 21.13 & 612.71 & 53.16 & 3.39 & 17.04 \\
\hline & \multirow{2}{*}{ oar-miR-150 } & QPCR & 0.01 & 0.01 & 0.03 & 1.00 & 1.03 \\
\hline & & FPKM & 1.27 & 3.73 & 13.77 & 3.39 & 17.04 \\
\hline & \multirow{2}{*}{ oar-miR-362 } & QPCR & 0.80 & 1.52 & 1.43 & 1.00 & 0.12 \\
\hline & & FPKM & 1376.64 & 2959.89 & 2391.22 & 879.08 & 354.87 \\
\hline & \multirow{2}{*}{ oar-miR-410-3p } & QPCR & 1.14 & 1.90 & 1.97 & 1.00 & 0.00 \\
\hline & & FPKM & 68.99 & 175.89 & 198.56 & 32.31 & 0.09 \\
\hline & \multirow{2}{*}{ oar-miR-221 } & QPCR & 1.49 & 1.29 & 1.65 & 1.00 & 1.67 \\
\hline & & FPKM & 46.88 & 52.56 & 80.03 & 34.52 & 91.31 \\
\hline \multirow{10}{*}{ LncRNA } & \multirow{2}{*}{ TCONS_00144061 } & QPCR & 28.44 & 28.18 & 28.73 & 1.00 & 0.88 \\
\hline & & FPKM & 22416.88 & 21299.49 & 25556.43 & 15182.36 & 10449.53 \\
\hline & \multirow{2}{*}{ TCONS_00105394 } & QPCR & 0.57 & 0.64 & 0.84 & 1.00 & 1.09 \\
\hline & & FPKM & 489.56 & 561.35 & 950.09 & 1454.33 & 2306.80 \\
\hline & \multirow{2}{*}{ TCONS_00105227 } & QPCR & 159.79 & 337.79 & 205.07 & 1.00 & 0.11 \\
\hline & & FPKM & 2137.33 & 2720.73 & 2231.06 & 423.75 & 66.20 \\
\hline & \multirow{2}{*}{ TCONS_00091985 } & QPCR & 0.26 & 0.49 & 0.80 & 1.00 & 1.18 \\
\hline & & FPKM & 546.08 & 557.27 & 590.00 & 1311.02 & 1469.90 \\
\hline & \multirow{2}{*}{ TCONS_00091984 } & QPCR & 0.05 & 0.04 & 0.03 & 1.00 & 0.72 \\
\hline & & FPKM & 148.81 & 230.36 & 202.90 & 987.67 & 572.94 \\
\hline
\end{tabular}

4 